\title{
A Non-interferometric Procedure for Deformation Measurement Using GB-SAR Imagery
}

\author{
M. Crosetto, O. Monserrat, G. Luzi, M. Cuevas, and N. Devanthéry
}

Abstract-Deformation monitoring using Ground-Based Synthetic Aperture Radar (GB-SAR) data usually exploits the interferometric phases. In this paper a new non-interferometric procedure is proposed, which exploits the geometric content of GB-SAR amplitude imagery and estimates deformation through image matching. This paper describes, step by step, this procedure. In order to achieve acceptable deformation measurement performances, the technique needs special targets, which have to guarantee a good image matching quality. If the available natural targets are insufficient, artificial corner reflectors are required. The new approach overcomes some of the main limitations of GB-SAR interferometry: it yields aliasing-free deformation estimates, it is insensitive to atmospheric effects, and it provides 2D displacement measurements while interferometry only has a mono-dimensional measurement capability. Several experiments focusing on the performances of the new procedure are described. The procedure was validated using different scenarios. On a real-size landslide scenario a mean absolute error over 12 corner reflectors of $0.59 \mathrm{~cm}$ was achieved, i.e. $1 / 85^{\text {th }}$ of the pixel size. These encouraging results can be of interest for several deformation monitoring applications.

Index Terms-Terrestrial SAR, SAR amplitude, corner reflectors, image matching, deformation.

\footnotetext{
Manuscript submitted for review November 28, 2012. This work was partially supported by the Working Community of the Pyrenees through the project SAXA (2010 CTP 00048).

M. Crosetto is with the Institute of Geomatics, Av. Gauss 11, Castelldefels, E-08860 Spain (phone: +34-93-556-92-94; fax: +34-93-556-92-92; e-mail: michele.crosetto@ideg.es).

O. Monserrat (e-mail: oriol.monserrat@ideg.es), G. Luzi (e-mail: guido.luzi@ideg.es), M. Cuevas (e-mail: maria.cuevas@ideg.es) and N. Devanthéry (email: nuria.devanthery@ideg.es) are with the Institute of Geomatics.
} 


\section{INTRODUCTION}

This paper describes a novel procedure to estimate deformation using GB-SAR observations. SAR is a relatively mature technology with numerous operational systems onboard satellites and aircrafts. In the last decade, it has been implemented on terrestrial platforms, offering a flexible deformation monitoring tool [1-3]. The GB-SAR has been used in a wide range of applications related to dams, landslides, subsidences, glaciers, volcanoes, avalanches and snow [4-6]. Displacement estimation with GB-SAR is usually based on the SAR interferometry technique GB-InSAR [7]. Thus, a coherent GB-SAR sensor, which measures both the phase and amplitude of the radar backscattered signal, is needed together with at least two SAR acquisitions of the same scene. Displacements between two acquisitions are derived from the interferometric phase, given by the difference between the phases acquired in two campaigns. The main advantage of this technique is its high sensitivity to small displacements: since most of the available GBSAR systems operate in the $1-5 \mathrm{~cm}$ wavelength region, a sub-millimetric displacement precision can usually be achieved for good targets. On the other hand, GB-InSAR suffers important limitations:

1. The conditio sine qua non for using interferometry is that a sufficient number of targets remain coherent over the observation period, which can last from weeks to months or years. The loss of temporal coherence can be particularly severe over vegetated areas [8].

2. A second critical limitation is aliasing. Due to the ambiguous nature of the interferometric phases, which vary between $[-\pi, \pi]$, the displacement undergone by a given scene can only unambiguously be estimated if the relative displacements actually occurring between adjacent coherent targets cause an interferometric phase difference smaller than $\pi$. In Ku-band $(\lambda=18 \mathrm{~mm})$ it corresponds to a maximum relative displacement of $4.5 \mathrm{~mm}$. From the operational point of view, this represents a severe limitation of the technique [9].

3. The atmospheric phase component caused by heterogeneous atmospheric conditions at the time of SAR image acquisitions is a third limitation that may degrade the quality of the estimation, and even affect the phase ambiguity estimation in the worst cases [9]. 
4. The last limitation is the mono-dimensional nature of the GB-InSAR measurements: given a generic 3D displacement, GB-InSAR can only estimate the radar Line-Of-Sight (LOS) component of the displacement.

The severity of the first three limitations, which also depends on the characteristics of the radar used (e.g. operating frequency), is largely affected by the GB-SAR measurement configuration used. The most common is the continuous mode, obtained by leaving the instrument installed in a given area during the entire period of interest [9]. In this case, the impact of the above limitations is usually moderate, if not negligible. In fact, with time lapses of minutes, coherence is usually very high, even over vegetated areas, and the aliasing and atmospheric effects are usually negligible. In contrast, this does not occur in the noncontinuous or discontinuous mode, where the instrument is used to revisit the same site over time, similarly to satellite acquisitions [10]. The discontinuous mode is appropriate to monitor slow displacement phenomena, where the continuous mode is usually not affordable. Its drawback is that all the above mentioned limitations play a major role.

This paper focuses on the discontinuous GB-SAR monitoring mode. A new deformation monitoring procedure that uses a GB-SAR non-interferometric technique, which potentially overcomes the last three limitations mentioned above, is proposed. This paper starts describing each step of the proposed procedure. Two key technical aspects, the image matching performances and the need of corner reflectors, are then discussed, followed by the analysis of several validation experiments and the conclusions.

\section{PROPOSED APPROACH}

The proposed procedure uses an image matching technique applied on amplitude GB-SAR images using special targets optimal for image matching purposes. The use of artificial Corner Reflectors (CRs) ensures obtaining optimal targets. Although CRs are not strictly necessary if a sufficient number of natural targets are available, the procedure described below assumes that only CRs are used. Two images of the same scenario are collected at different times (master and slave). We focus on pairs of common or homologous 
points identified in both images: $P$ and $P^{\prime}$. The image displacement vector $\left(\mathrm{S}_{\mathrm{x}}, \mathrm{S}_{\mathrm{y}}\right)$ between $P$ and $P^{\prime}$ is estimated by means of an image matching technique working at sub-pixel level. $\left(\mathrm{S}_{\mathrm{x}}, \mathrm{S}_{\mathrm{y}}\right)$ is then transformed into a 2D deformation vector, which measures the displacement in the range and cross-range directions. The potential 2D deformation measurement capability represents an interesting advantage with respect to the GB-InSAR LOS measurement. A second fundamental advantage is the aliasing-free deformation measurement. Finally, another advantage is that this estimation is basically insensitive to the atmospheric effects.

Image matching is widely exploited to measure deformation using satellite SAR amplitude imagery [11]. The most innovative aspect of this procedure is the proposal of a method to achieve reliable and precise deformation estimates. The second part of the paper discusses the performances of the technique in detail. Fig. 1 shows an example of results generated with the proposed procedure, which concerns the landslide of Vallcebre (Eastern Pyrenees, Spain). In this case, displacement vectors ranging between $7.1 \mathrm{~cm}$ and 11.2 cm were retrieved using only two campaigns. It is literally impossible to obtain similar results with GBInSAR, unless a much higher number of campaigns is used to avoid aliasing.

The core of the procedure is three-fold: (i) employing a GB-SAR in a discontinuous mode; (ii) using image matching procedures; and (iii) deploying CRs. These are, per se, known and established techniques. The innovative aspect of the procedure consists of using these components jointly and in an optimized way for displacement monitoring purposes, thus achieving interesting measurement performances as shown later in this paper. The main steps of the proposed procedure are:

- Data acquisition: this is obtained through a series of $N$ in-situ campaigns. Each campaign consists of installing the GB-SAR instrument, deploying a set of $M$ CRs in the area of interest and acquiring $K$ complex SAR images. GB-SAR positioning is usually performed in a relatively light fashion, e.g. without building a stable concrete base, making the data acquisition easier. 
- Data pre-processing: this step is performed on each set of $K$ SAR images, and hence is repeated $N$ times. It involves checking the quality of each acquired image. A temporal filtering is then performed on the sub-set of quality-proofed images, which includes $Q \leq K$ images, whose goal is image enhancement by reducing the speckle effect. This is done through incoherent temporal averaging of the $Q$ images, obtaining $A_{\text {incoh }}^{k}$.

- Global matching: this step is performed in each pair $i j$ of campaigns. Given $N$ campaigns, the minimum number of global matching is $N$-1. Its input is a pair of incoherently averaged images: $A_{\text {incoh }}^{i}$ and $A_{\text {incoh. }}^{j}$ A pixel selection is then performed, whose goal is identifying suitable pixels for image matching, which include those associated with the $M$ deployed CRs and other natural reflectors, like exposed rocks, buildings, etc. The global image matching is performed over the set of selected pixels. Although several algorithms can be used for this purpose [12-13], the results described in this paper were obtained using the incoherent cross-correlation based on the amplitude images. An additional selection is then performed in the outcome of the global matching, i.e. $W$ pairs of global shifts, using the crosscorrelation coefficient. The result of the global matching is finally a selected set of $R \leq W$ pairs of global shifts.

- Estimation of the GB-SAR repositioning effects: this is performed on each analysed pair ij of campaigns. These effects are caused by slight changes in the position and orientation of the GB-SAR at each campaign. The estimation of these effects involves the pre-selection of the stable areas which can be $a$ priory known or just detected as stable by using a statistical approach. A subset of the above $R$ pairs of global shifts, given by the $S \leq R$ global shifts that fall in the stable area, is computed. The $S$ global shifts are used to estimate the co-registration transformation, whose complexity mainly depends on the topography and the sensor to target distance. The author's experience suggests that, excluding closerange scenarios (e.g. at less than $100 \mathrm{~m}$ ) and strong topographic variations, the affine transformation is usually sufficient. The final result is a set of co-registration transformation parameters. 
- Estimation of the pairs of displacements: this step is performed on each analysed pair ij of campaigns by subtracting the GB-SAR-repositioning effects from the set of $R$ pairs of global shifts. It results in $R$ pairs of displacement shifts that are relative to the whole set of stable areas mentioned above. The above displacement shifts are then transformed into displacements. Finally, considering the position and orientation of the GB-SAR sensor, image geocoding is performed obtaining, for each measured point, its cartographic or geographic coordinates.

- Estimation of the displacement time series: This step has to be carried out once for all campaigns to obtain the main product of the procedure: the estimated displacement time series. It involves obtaining, for each analysed pair ij of campaigns, a set of $R_{i j}$ pairs of estimated displacements defined in the object space. The displacements are estimated by Least Squares (LS):

$D_{i j}=D_{j}-D_{i}+\varepsilon$

$D_{0}=0$

where $D_{i}$ and $D_{j}$ are the unknown accumulated displacements at time (i) and (j), and $\varepsilon$ are the residuals [14]. A redundant configuration is assumed, i.e. multiple pairs for each measurement, to attain a certain degree of displacement controllability. The LS estimation can be used to identify and isolate outliers in the input data, i.e. in the $D_{i j}$ pairs, and this process can be iterative. The final product is a set of $D$ estimated displacement time series.

\section{DISCUSSION OF THE PROPOSED METHOD}

This section discusses two fundamental technical aspects of the proposed procedure: image matching performances and the need of deploying CRs. An interesting property of the used image matching procedure is shown in Fig. 2. The bold line shows the theoretical relation between the matching precision (called matching shift error in [13]) and the Signal-to-Clutter Ratio (SCR), which is useful to estimate the expected matching precision from the SCR of a give target. The validity of this relation was studied empirically using real GB-SAR data. The peak-to-background ratio (PBR) was computed dividing the 
intensity of a given point scatterer by the intensity of its neighbours, located in a 16 by 16 pixel window: the PBR is considered as an approximation of the SCR in this work. Three experiments were performed (Cal Ganxo, Vallcebre and the Canal Olímpic) using the IBIS-L Ku-band GB-SAR, manufactured and marketed by IDS SpA, using an image pixel spacing in range of $0.5 \mathrm{~m}$ and a cross-range angular spacing of 4.4 mrad. All the experiments were based on triangular and square trihedral CRs of different size, ranging from $20 \mathrm{~cm}$ (close-range) to $50 \mathrm{~cm}$ (up to $1600 \mathrm{~m}$ ). The image matching was performed on 16 by 16 pixel windows. The sequence of the three experiments involved the following steps: (i) positioning the GB-SAR and acquiring a set of 15 images; (ii) dismounting the GB-SAR and re-mounting it after a short lapse of time; (iii) acquiring a second set of 15 images. The short lapse of time between image acquisitions allowed assuming that deformation-free scenarios were observed; (iv) applying the proposed procedure over a set of selected targets including both natural reflectors and CRs; (v) analysing the estimated deformation for each target, considering that no displacements were expected for all targets. A total amount of 60247 targets were analysed, discarding the targets with a cross-correlation coefficient lower than 0.6. Fig. 2 shows the plots of the matching precision as a function of the PBR. The black diamonds represent the reference theoretical values from [13], while the other lines represent the experimental results. Two main conclusions can be derived:

- The experimental results in range agree with the theoretical ones. A matching precision of about 0.02 pixels, which corresponds to $1 \mathrm{~cm}$ for the used GB-SAR sensor, can be achieved on targets with PBR > $30 \mathrm{~dB}$.

- The results in cross-range are sensibly worse. In the best case scenario (PBR > 30dB), the precision ranges from 0.02 to 0.07 pixels, i.e. $8.8 \mathrm{~cm}$ to $30.8 \mathrm{~cm}$ at $1 \mathrm{~km}$. These numbers show remarkable different performances of the technique in range and cross-range directions.

An additional significant result is that the number of natural targets with a sufficiently high PBR to guarantee good deformation estimates is rather low. For the Cal Ganxo experiment, which includes an 
urban area, the targets with PBR higher than $30 \mathrm{~dB}$ are 73 , i.e. the $0.32 \%$ of the measured pairs. Similar values were obtained in the Canal Olímpic experiment, $0.28 \%$, and in the Vallcebre experiment, $0.27 \%$. In the latter case, which concerns an active landslide, there are no good targets in the deformation area. In general, these percentages are insufficient to obtain an appropriate spatial sampling of deformation phenomena. This issue clearly demonstrates the need of deploying CRs. In this regard, CRs are the only guarantee of obtaining a good and persistent target response over time in a given area of interest, which results in good deformation measurements. The drawback is the need of substitute a truly remote sensing procedure with a hybrid procedure, which is supported by in-situ installed CRs.

\section{VALIDATION RESULTS}

Five validation experiments were carried out to test the procedure in different conditions and scenarios. They were carried out with the IBIS-L Ku-band GB-SAR, using a pixel spacing in range of $0.5 \mathrm{~m}$ and a cross-range angular spacing of $4.4 \mathrm{mrad}$. In two of them, the Campus experiments, the performances of the proposed technique were assessed working in close-range conditions. The Beach experiment was performed using CRs of different sizes located at different ranges. The Cal Ganxo experiment involved CRs located at far distances, up to $1650 \mathrm{~m}$. An additional validation experiment was the Vallcebre landslide. Two types of experiments were performed. Artificially-induced deformations were obtained by imposing known displacements to a set of CRs in the first four experiments. For the landslide, independent ground truth collected during surveying campaigns was used. The estimated GB-SAR range displacements were compared with the reference values measured with an electronic distance meter located at the same position of the GB-SAR. The reference values are characterized by a standard deviation below $2 \mathrm{~mm}$.

Table 1 shows the results from the Campus experiments. Considering the range validation errors, i.e. the difference between the GB-SAR estimates and the reference values, 15 out of 20 CRs estimated are below 1 $\mathrm{cm}$, i.e. below $1 / 50^{\text {th }}$ of range pixel spacing, two errors are between 1 and $2 \mathrm{~cm}$, and the remaining three are outliers. These outliers are associated with targets with low PBR: 20, 28 and $21 \mathrm{~dB}$, which confirm the results discussed in the previous section. The cross-range results are sensibly worse. Considering the nine 
CRs with PBR above $30 \mathrm{~dB}$, three of the cross-range validation errors are below $2 \mathrm{~cm}$, while most of them are of several centimetres. The error size has no correlation with the magnitude of displacements. The above results are slightly worse than those from Fig. 2, which indicate a precision ranging from $2 \%$ to $7 \%$ of the cross-range pixel size: five of the nine CRs considered above have errors considerably larger than $7 \%$. Further work is needed to improve the quality of these deformation estimates. However, these results show the 2D capability of the proposed technique. Table 2 summarizes the results of the remaining three experiments, which only focus on the range direction. In the Beach experiment, six of the eight CRs with PBR above $30 \mathrm{~dB}$ have validation errors well below $1 \mathrm{~cm}$, one has an error of $1.11 \mathrm{~cm}$ and another one shows a gross error. This error is explained by the distribution of the CRs in the scene: the matching window of the corresponding CR was affected by the side lobes of another CR which was moved in a different way. In the Cal Ganxo experiment, the four CRs with high PBR have validation errors below $1 \mathrm{~cm}$. Note that all of them were located at more than $1550 \mathrm{~m}$ from the GB-SAR. In contrast, the three CRs with PBR below 30 dB show large errors. In the Vallcebre landslide, the validation results were derived by comparison with ground truth. All the 12 CRs, ranging from 507 to $832 \mathrm{~m}$ and with high PBR (mean PBR = $34 \mathrm{~dB}$ ), show validation errors below $1 \mathrm{~cm}$ (max. absolute value $=0.88 \mathrm{~cm}$, mean $=0.59 \mathrm{~cm}$ ). It is worth noting that these values include the topographic measurement uncertainty. They are very encouraging from both the point of view of image matching $\left(0.59 \mathrm{~cm}\right.$ corresponds to $1 / 85^{\text {th }}$ of the pixel size $)$ and the deformation measurement.

\section{CONCLUSIONS}

In this paper, a new GB-SAR deformation measurement procedure has been proposed. It requires special targets, optimal for image matching purposes, to achieve good results, which can be obtained by using artificial CRs. A complete description of the proposed procedure has been provided. It overcomes some of the main limitations of GB-InSAR, which are particularly critical in the discontinuous GB-SAR monitoring mode. Its drawback is a reduction of the sensitivity to deformation and a reduced deformation estimate precision, which ranges from a few millimetres to $1 \mathrm{~cm}$. Depending on the application at hand, one can choose between the GB-InSAR, which is more precise but prone to aliasing errors, or the new approach, 
which is less sensitive but more robust. Both approaches are compatible because they use the same input data. The proposed procedure is insensitive to the atmospheric effects. The method provides 2D displacement measurements in range and cross-range directions. However, the current procedure shows rather weak performances in the cross-range direction, even at close distances. Further work is needed to obtain a fully 2D deformation monitoring technique.

The procedure has been successfully validated in different scenarios. The validation results confirm the poor performances of the deformation estimates in cross-range, and the good results in range (errors below 0.02 pixels) with PBR higher than $30 \mathrm{~dB}$. The Vallcebre results are particularly encouraging, with mean absolute error over $12 \mathrm{CRs}$ of $0.59 \mathrm{~cm}\left(1 / 85^{\text {th }}\right.$ of the pixel size). These numbers are surely interesting for several deformation monitoring applications. A practical limitation is related to the size of the CRs, which, in order to guarantee a good PBR, has to increase with the sensor-to-target distance.

Further research is needed to improve some aspects of the procedure. The condition of $30 \mathrm{~dB}$ on the PBR need to be relaxed in order to increase its applicability: new image matching algorithms are needed. A second aspect is related to the size of the CRs, which has to be increased considerably in order to keep high PBR values, especially at long distances, e.g. above $2 \mathrm{~km}$. The use of active CRs would drastically reduce the CR size, thus improving the measurement logistics. 


\section{REFERENCES}

[1] D. Tarchi, N. Casagli, R. Fanti, D. Leva, G. Luzi, A. Pasuto, M. Pieraccini, and S. Silvano, "Landslide monitoring by using ground-based SAR interferometry: an example of application to the Tessina landslide in Italy,” Eng. Geol., vol. 68, pp.15-30, Feb. 2003.

[2] M. Pieraccini, N. Casagli, G. Luzi, D. Tarchi, D. Mecatti, L. Noferini, and C. Atzeni, "Landslide monitoring by ground-based radar interferometry: a field test in Valdarno (Italy)”, Int. J. Remote Sens., vol. 24(6), pp. 1385-1391, Mar. 2003.

[3] L. Pipia, X. Fabregas, A. Aguasca, C. López-Martínez, S. Duque, J. J. Mallorqui, J. Marturia, “Polarimetric differential SAR interferometry: first results with ground-based measurements," IEEE Geosci. Remote S., vol. 6(1), pp. 167-171, Jan. 2009.

[4] G. Herrera, J. A. Fernandez-Merodo, J. Mulas, M. Pastor, G. Luzi, and O. Monserrat, “A landslide forecasting model using ground based SAR data: the Portalet case study,” Eng. Geol., vol. 105(3-4), pp. 220-230, May 2009.

[5] S. Rödelsperger, G. Läufer, C. Gerstenecker, and B. Matthias, "Monitoring of displacements with ground-based microwave interferometry,: IBIS-S and IBIS-L,” Journal of Applied Geodesy, vol. 4, pp. 41-54, Jun. 2010.

[6] G. Luzi, M. Pieraccini, D. Mecatti, L. Noferini, G. Macaluso, A. Tamburini, and C. Atzeni, "Monitoring of an Alpine Glacier by Means of Ground-Based SAR Interferometry,” IEEE Geosci. Remote S., vol. 4(3), pp. 495-499, Jul. 2007.

[7] G. Luzi, M. Pieraccini, D. Mecatti, L. Noferini, G. Guidi, F. Moia, and C. Atzeni, “Ground-based radar interferometry for landslides monitoring: Atmospheric and instrumental decorrelation sources on experimental data,” IEEE T. Geosci. Remote, vol. 42(11), pp. 2454-2466, Nov. 2004. 
[8] L. Noferini, M. Pieraccini, D. Mecatti, G. Luzi, A. Tamburini, M. Broccolato, and C. Atzeni, “Permanent scatterers analysis for atmospheric correction in Ground Based SAR Interferometry,” IEEE T. Geosci. Remote, vol. 43(7), pp. 1459-1471, Jul. 2005.

[9] O. Monserrat, “Deformation measurement and monitoring with Ground-Based SAR,” Ph.D. dissertation, Institute of Geomatics, Publication N. 1, 2012. Available on-line at www.ideg.cat.

[10] G. Luzi, O. Monserrat, M. Crosetto, R. Copons, and J. Altimir, "Ground-Based SAR interferometry applied to landslide monitoring in mountainous areas," in Proc. Mountain Risks Int. Conf., Florence (Italy), 24-26 November 2010, pp. 307-312.

[11] T. J. Wright, C. Ebinger, J. Biggs, A. Ayele, G. J. Yirgu, D. Keir, and A. Stork, “Magma-maintained rift segmentation at continental rupture in the 2005 Afar dyking episode,” Nature, vol 442, 291-294, doi:10.1038/nature04978, Jul. 2006.

[12] A. Gruen, “Adaptive least squares correlation - A powerful image matching technique,” South African Journal of Photogrammetry, Remote Sensing and Cartography, vol. 14, pp. 175-187, 1985.

[13] R. Bamler, and M. Eineder, "Split Band Interferometry versus absolute ranging with wideband SAR systems,” in Proc. IGARSS 2004, Anchorage (USA), 2004, vol. 2, pp. 980-984.

[14] O. Monserrat, M. Crosetto, and B. Crippa, and G. Herrera, “A 2+1D phase unwrapping for advanced DInSAR data processing,” IEEE T. Geosci. Remote, submitted for publication. 


\section{TABLES}

Table 1. Validation results from the two simulated Campus experiments.

\begin{tabular}{|c|c|c|c|c|c|c|c|c|c|}
\hline & $\begin{array}{l}\text { CR } \\
\mathrm{N}^{\circ}\end{array}$ & \begin{tabular}{|c} 
Sensor \\
target \\
distance \\
{$[\mathrm{m}]$} \\
\end{tabular} & \begin{tabular}{|c|} 
Reference \\
Range \\
displacement \\
{$[\mathrm{cm}]$} \\
\end{tabular} & \begin{tabular}{|c|} 
Estimated \\
Range \\
displacement \\
{$[\mathrm{cm}]$} \\
\end{tabular} & \begin{tabular}{|c|} 
Reference \\
Cross-Range \\
displacement \\
{$[\mathrm{cm}]$} \\
\end{tabular} & $\begin{array}{c}\text { Estimated } \\
\text { Cross-Range } \\
\text { displacement } \\
{[\mathrm{cm}]} \\
\end{array}$ & $\begin{array}{r}\text { Range } \\
\text { Error } \\
{[\mathrm{cm}]}\end{array}$ & \begin{tabular}{|c|} 
Cross \\
Range \\
Error \\
{$[\mathrm{cm}]$} \\
\end{tabular} & $\begin{array}{l}\text { PBR } \\
\text { [dB] }\end{array}$ \\
\hline \multirow{9}{*}{ 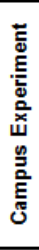 } & 2 & 64.5 & -1.00 & \begin{tabular}{|l|}
-1.55 \\
\end{tabular} & -1.00 & -0.88 & 0.55 & -0.12 & 31 \\
\hline & 10 & 70.0 & -5.00 & -5.45 & -5.00 & -8.65 & 0.45 & 3.65 & 31 \\
\hline & 3 & 80.0 & -10.00 & -10.95 & -10.00 & -16.51 & 0.95 & 6.51 & 30 \\
\hline & 4 & 119.5 & -10.00 & -18.75 & 0.00 & 0.00 & 8.75 & 0.00 & 20 \\
\hline & 5 & 127.0 & -15.00 & -16.40 & 0.00 & 0.00 & 1.40 & 0.00 & 28 \\
\hline & 9 & 131.5 & -20.00 & -7.00 & 0.00 & -7.23 & -13.00 & 7.23 & 28 \\
\hline & 8 & 133.5 & 0.00 & 0.00 & 0.00 & 0.00 & 0.00 & 0.00 & 27 \\
\hline & 1 & 211.5 & -20.00 & -6.25 & 0.00 & -17.50 & -13.75 & 17.50 & 21 \\
\hline & 6 & 220.0 & 0.00 & -0.75 & 0.00 & 0.00 & 0.75 & 0.00 & 26 \\
\hline \multirow{11}{*}{ 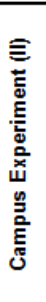 } & 2 & 79.5 & -12.00 & -12.50 & -14.00 & -18.05 & 0.50 & 4.05 & 32 \\
\hline & 1 & 80.0 & 0.00 & 0.00 & 0.00 & -1.20 & 0.00 & 1.20 & 34 \\
\hline & 3 & 91.0 & -20.00 & -20.30 & -20.00 & -23.39 & 0.30 & 3.39 & 16 \\
\hline & 4 & 110.5 & -80.00 & -79.70 & 0.00 & -1.66 & -0.30 & 1.66 & 29 \\
\hline & 5 & 134.0 & 0.00 & 0.00 & 0.00 & 0.00 & 0.00 & 0.00 & 34 \\
\hline & 6 & 167.5 & 30.00 & 28.10 & 0.00 & -5.03 & 1.90 & 5.03 & 27 \\
\hline & 11 & 179.5 & 15.00 & 15.60 & -40.00 & -46.13 & -0.60 & 6.13 & 32 \\
\hline & 10 & 194.0 & -40.00 & -39.05 & 0.00 & -2.91 & -0.95 & 2.91 & 31 \\
\hline & 7 & 201.0 & 0.00 & 0.00 & 0.00 & -9.14 & 0.00 & 9.14 & 32 \\
\hline & 9 & 215.0 & 0.00 & 0.00 & 0.00 & -9.78 & 0.00 & 9.78 & 29 \\
\hline & 8 & 232.5 & 35.00 & 34.40 & 0.00 & 3.49 & 0.60 & -3.49 & 27 \\
\hline
\end{tabular}


Table 2. Validation results from three experiments: Beach, Cal Ganxo and the real deformation scenario of the Vallcebre landslide.

\begin{tabular}{|c|c|c|c|c|c|c|}
\hline & CR name & $\begin{array}{c}\text { Sensor } \\
\text { target } \\
\text { distance } \\
\text { [m] }\end{array}$ & \begin{tabular}{|c|} 
Reference \\
Range \\
displacement \\
[cm] \\
\end{tabular} & $\begin{array}{c}\text { Estimated } \\
\text { Range } \\
\text { displacement } \\
\text { [cm] } \\
\end{array}$ & $\begin{array}{c}\text { Error } \\
\text { [cm] }\end{array}$ & $\begin{array}{r}\text { PBR } \\
\text { [dB] }\end{array}$ \\
\hline \multirow{10}{*}{ 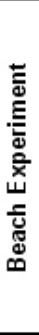 } & Stmall 1 & 223 & 26.10 & 25.78 & 0.32 & 33 \\
\hline & Small 2 & 239 & 9.00 & 9.38 & -0.38 & 34 \\
\hline & Small 3 & 230 & -20.10 & -20.31 & 0.21 & 31 \\
\hline & Small 4 & 230 & -17.30 & -18.75 & 1.45 & 26 \\
\hline & Medium 1 & 575 & 18.00 & 18.75 & -0.75 & 35 \\
\hline & Medium 2 & 597 & 28.80 & 28.13 & 0.68 & 30 \\
\hline & Big 1 & 964 & -19.10 & -19.50 & 0.40 & 32 \\
\hline & Big 2 & 1039 & 19.20 & 20.31 & -1.11 & 32 \\
\hline & Big 3 & 1036 & -30.80 & -14.06 & -16.74 & 32 \\
\hline & Big 4 & 1080 & 23.50 & 21.90 & 1.60 & 21 \\
\hline \multirow{7}{*}{ 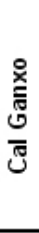 } & 1 & 1521 & -15.00 & 1.55 & -1655 & 26 \\
\hline & 2 & 1504 & 15.00 & 10.00 & 5.00 & 27 \\
\hline & 3 & 1531 & -15.00 & -60.00 & 45.00 & 21 \\
\hline & 4 & 1557 & -30.60 & -30.00 & -0.60 & 35 \\
\hline & 5 & 1573 & 22.50 & 23.45 & -0.95 & 34 \\
\hline & 6 & 1604 & -27.00 & -26.55 & -0.45 & 33 \\
\hline & 7 & 1658 & -14.70 & -14.05 & -0.65 & 35 \\
\hline \multirow{12}{*}{ 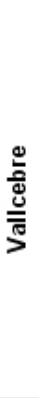 } & CR01 & 507 & -9.77 & -8.88 & -0.88 & 34 \\
\hline & $\mathrm{CR} 03$ & 533 & -10.62 & -10.05 & -0.57 & 34 \\
\hline & $\mathrm{CR} 05$ & 544 & -10.62 & -10.05 & -0.57 & 34 \\
\hline & CR06 & 600 & -9.02 & -9.67 & 0.65 & 35 \\
\hline & CR09 & 446 & -8.52 & -8.88 & 0.36 & 34 \\
\hline & CR10 & 441 & -8.02 & -7.16 & -0.86 & 34 \\
\hline & CR11 & 483 & -9.92 & -10.44 & 052 & 35 \\
\hline & CR12 & 531 & -11.42 & -11.22 & -0.20 & 34 \\
\hline & CR14 & 524 & 0.70 & 0.88 & -0.18 & 34 \\
\hline & CR15 & 708 & 0.80 & 0.10 & 0.70 & 36 \\
\hline & CR16 & 467 & 0.90 & 0.10 & 0.80 & 34 \\
\hline & CR17 & 833 & -1.82 & -1.07 & -0.75 & 31 \\
\hline
\end{tabular}


FIGURES

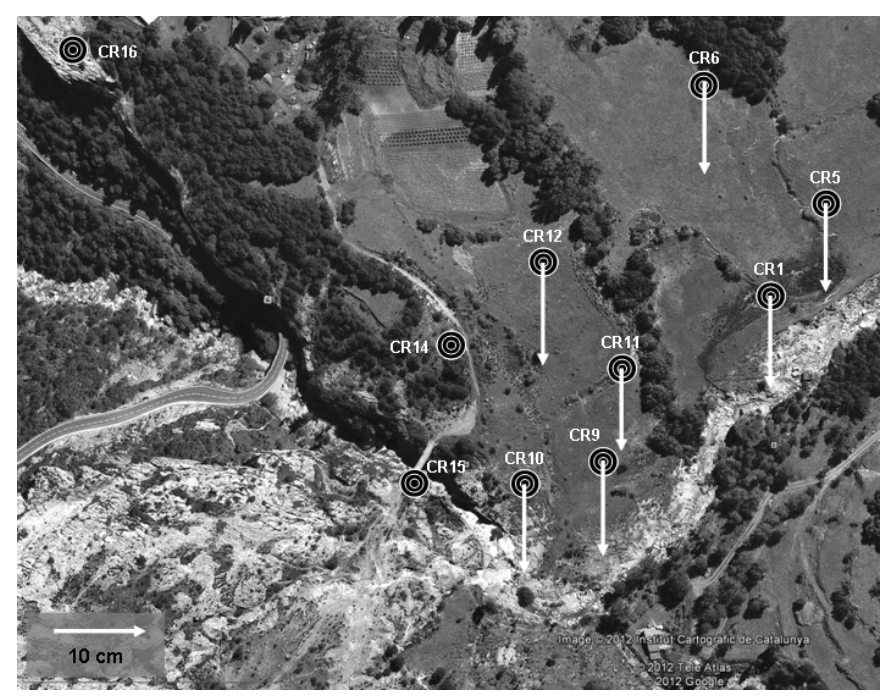

Fig. 1. Displacements of the Vallcebre landslide in the range direction estimated using two GB-SAR campaigns with a time lapse of 4 months. 

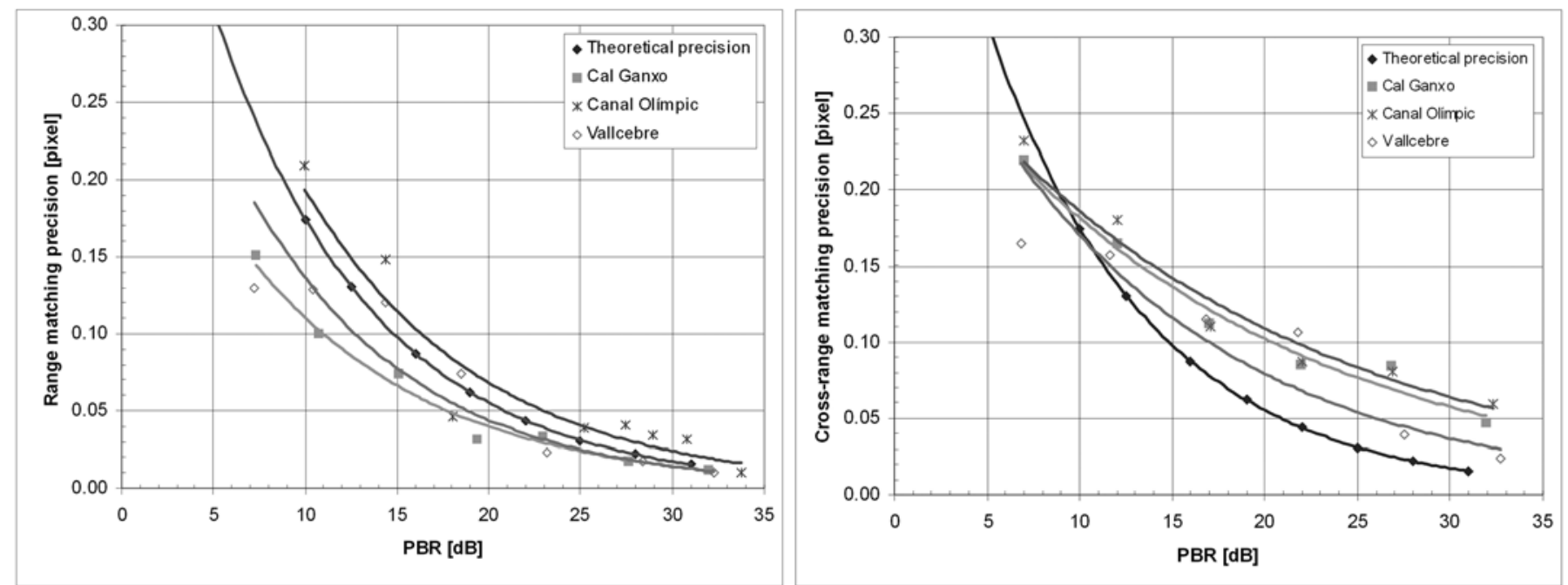

Fig. 2. Theoretical and experimental matching precision in the range (left) and cross-range (right) directions, respectively, as a function of the Peak-to-Background Ratio (PBR). The black line also represents the matching precision of a point scatterer as a function of the signal-to-clutter ratio [13]. 